\title{
Assessment of Direct Oral Anticoagulant Use at a Community Teaching Hospital
}

Alaina Van Dyke, PharmD, MBA ${ }^{1}$ and Doug Carroll, PharmD, BCPS ${ }^{2}$

Department of Pharmacy Practice, Jerry H. Hodge School of Pharmacy, Texas Tech University Health Sciences Center ${ }^{1}$;

DCH Regional Medical Center ${ }^{2}$

\begin{abstract}
Direct oral anticoagulants (DOACs) have become popular choices for both the treatment and prevention of thromboembolic events. However, these agents pose additional risks to patients due to complex dosing, insufficient monitoring, and inconsistent patient compliance. This study evaluates the appropriateness of DOAC prescribing for patients who received an order for apixaban or rivaroxaban over a 6-month period. The primary outcome is percentage of inappropriately prescribed DOAC regimens. Secondary outcomes include an effectiveness endpoint of stroke or embolism and a safety endpoint of major bleeding documented during or within 60 days of the initial visit as well as number of pharmacist clinical interventions. DOAC orders were appropriate $73 \%$ of the time. Of the $27 \%$ of inappropriate orders, approximately half were apixaban and half were rivaroxaban. The most common reason for an inappropriate order for apixaban was due to atrial fibrillation dosing, and the most common reason for an inappropriate rivaroxaban order was due to dose-indication mismatch. There were 30 pharmacist clinical interventions on DOAC orders that were documented during the 6-month period, and the most common reason for a pharmacist intervention was duplication with another anticoagulant.
\end{abstract}

Keywords: DOACs, anticoagulation, prescribing habits

\section{Introduction}

Over the past decade, direct oral anticoagulants (DOACs) have become popular choices for both the treatment and prevention of thromboembolic events and are now even preferred over the traditional vitamin $\mathrm{K}$ antagonist for certain indications [1]. However, according to The Joint Commission's 2019 Hospital National Patient Safety Goals (NPSGs), these agents pose additional risks to patients due to "complex dosing, insufficient monitoring, and inconsistent patient compliance [2]." Appropriate dosing of these agents is important to prevent underdosing, which could lead to thromboembolic events or strokes, or overdosing, which may result in bleeding events.

DOAC dosing can be confusing for a few reasons. First, the dosing regimens are different for each drug based on indication (treatment of VTE, prophylaxis of VTE, or AF). Secondly, these agents often require dose adjustments based on patientspecific parameters, such as renal function, age, weight, etc., which again is different depending on the drug and indication. Several recent studies have looked into the dosing patterns for these agents. Yoa et al evaluated DOAC dosing in nearly 15,000 patients with atrial fibrillation (AF) and found that approximately $43 \%$ of patients with AF and renal impairment were potentially overdosed, leading to higher incidents of bleeding. The study also found that $13 \%$ of patients with AF and no renal impairment were potentially underdosed, and this was associated with higher risk of stroke [3]. Miele et al found that a pharmacist driven DOAC service was associated with a significant decrease in inappropriate DOAC prescribing [4].

Corresponding author: Doug Carroll, PharmD, BCPS

DCH Regional Medical Center

Email: douglas.carroll@dchsystem.com
A previous review of DOACs at DCH suggested that prescribing was inappropriate for apixaban about $50 \%$ of the time. Furthermore, review of pharmacy clinical interventions specific to anticoagulants for a 90-day timeframe suggest that there is still a high incidence of inappropriate DOAC prescribing. Due to the increased use of these medications, their preference being emphasized in guidelines, and NPSGs surrounding these agents, further evaluation should be conducted to determine whether education or other interventions need to be implemented at DCH $[1,2]$.

\section{Materials and Methods Setting}

This performance improvement project took place at $\mathrm{DCH}$ Regional Medical Center, a 583-licensed bed, non-profit, community teaching hospital with a decentralized pharmacy department. Currently, decentral pharmacists are consulted by prescribers to dose warfarin and are responsible for warfarin patient education; however, there is currently little pharmacist involvement in the management and education of DOACs which are extensively used at this institution.

\section{Outcomes}

The primary outcome of this project was percentage of inappropriately prescribed DOAC regimens. Appropriateness was defined according to dosing recommendations in the products' package insert [5-8]. An order was considered inappropriate if it was dosed incorrectly based on the indication or renal function, was used for an indication that is not listed in the package insert, or was used concomitantly with another anticoagulant. Secondary outcomes included an effectiveness endpoint of stroke or embolism documented during or within 60 days of the initial visit and a safety endpoint of major bleeding also documented during or within 60 days of the initial visit. Major bleeding events were defined according to the International Society of Thrombosis and Haemostasis (ISTH) criteria as clinically 
overt bleeding accompanied by at least one of the following: bleed leading to fatal outcome, a decrease in hemoglobin level of at least $2 \mathrm{~g} / \mathrm{dL}$, transfusion of at least two units of packed red blood cells, or involvement of a critical site (intracranial, spinal, ocular, pericardial, articular, retroperitoneal or intramuscular with compartment syndrome) [9].

\section{Study Design and Sample}

This project utilized retrospective chart review to gather deidentified patient data to determine appropriateness of DOAC prescribing. The study sample consists of patients 19 years of age and older who were admitted to $\mathrm{DCH}$ for any reason and received an order for either apixaban or rivaroxaban for any indication during the months of January through June 2019. The authors generated a patient-on-drug list in Meditech, the hospital's electronic medical record (EMR), to provide a report of the patients mentioned above. Every other patient on the list was included in data collection to obtain a sample size of 100 patients (50 for each drug). Since the most frequently occurring indication was likely to be post-op VTE prophylaxis, and to ensure that a variety of indications are included in the analyses, an indication of post-op VTE prophylaxis was limited to 10 patients for each drug. The authors also generated a pharmacy clinical intervention report through Meditech. This list was screened for any interventions that were made regarding apixaban and rivaroxaban during the same 6-month time frame.

The following data was collected: age, gender, weight, serum creatinine, creatinine clearance, anticoagulant dose, frequency, and indication, whether it is being used as a home medication, use of other anticoagulant or antiplatelet, bleeding event, or thrombotic event. Prescriber specialty (cardiologist, hospitalist, nephrologist, orthopedist, other), documented pharmacist clinical interventions, and DOAC discharge regimen were also collected. Lastly, any changes in renal function during the course of the initial visit (while on DOAC therapy) that warranted a dose adjustment was documented. Patients were only reviewed for this if their initial creatinine clearance was within $15 \mathrm{ml} / \mathrm{min}$ of the dosing cut-off and a minimum change in serum creatinine of $0.3 \mathrm{mg} / \mathrm{dL}$ was noted during the course of the initial visit.

\section{Results}

Descriptive Analysis

A total of 130 patients were included in this review. Onehundred patients were included in the primary endpoint, and 30 patients were included in the pharmacist clinical intervention review. Table 1 highlights baseline characteristics, which were similar between groups.

For the primary endpoint (Figure 1), 73\% of DOAC orders were appropriate. Of the $27 \%$ of inappropriate orders, 14 (52\%) were apixaban orders and 13 (48\%) were rivaroxaban orders. Table 2 highlights inappropriate orders by indication. To explain this further, there were a total of 50 orders for both apixaban and rivaroxaban. Of the 50 apixaban orders, 14 (28\%) were inappropriate. There were a total of 13 apixaban orders that were specifically for the treatment of an active venous thromboembolism (VTE). Of those 13 orders, 5 (38\%) were inappropriate for some reason.

Table 1. Baseline Characteristics

\begin{tabular}{|lcc|}
\hline & Apixaban & Rivaroxaban \\
\hline Gender (male, \%) & $\mathrm{n}=50$ & $\mathrm{n}=50$ \\
Mean Age (years) & 44 & 56 \\
DOAC home medication (\%) & $69( \pm 12.1)$ & $65( \pm 13.4)$ \\
\hline
\end{tabular}

Figure 1.

Assessment of DOAC Prescribing

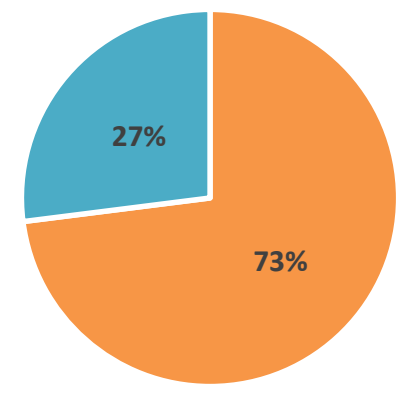

- Appropriate Inappropriate 
Table 2. Inappropriate Prescribing by Indication

\begin{tabular}{|lcc|}
\hline & Apixaban & Rivaroxaban \\
\hline Overall & $14 / 50(28 \%)$ & $13 / 50(26 \%)$ \\
VTE Treatment & $5 / 13(38 \%)$ & $0 / 5(0 \%)$ \\
VTE Risk Reduction & $2 / 2(100 \%)$ & $7 / 13(54 \%)$ \\
Stroke Prevention in AF & $7 / 26(27 \%)$ & $5 / 22(23 \%)$ \\
Post-op Prophylaxis & $0 / 9(0 \%)$ & $1 / 10(10 \%)$ \\
\hline
\end{tabular}

Figures 2 and 3 show a breakdown of reasons for inappropriate orders for apixaban and rivaroxaban respectively. The most common reason for an inappropriate apixaban order (Figure 2) was incorrect dosing for atrial fibrillation (AF), which is based on 3 criteria specific to this indication. Most patients receiving apixaban for stroke prevention in the setting of AF will receive a dose of $5 \mathrm{mg}$ to be taken twice daily. However, if the patient meets any 2 out of the 3 criteria, he/she should receive a dose of $2.5 \mathrm{mg}$ to be taken twice daily. The AF dosing criteria are as follows: age $\geq 80$ years old, weight $\leq 60 \mathrm{~kg}$, and serum creatinine $\geq 1.5 \mathrm{mg} / \mathrm{dL}$.

The second most common reason for an inappropriate apixaban order was dose-indication mismatch, which simply means that the dose of the drug was inappropriate based on the indication for which it was being used. Lastly, some orders were inappropriate due to duplication with another anticoagulant such as a heparin product, warfarin, or even another DOAC.

Unlike apixaban, rivaroxaban does not have specific criteria for AF dosing. However, it does have specific dosing adjustments based on the patient's renal function. For example, the dose of rivaroxaban for stroke prevention in the setting of $A F$ is $20 \mathrm{mg}$ daily for a patient whose creatinine clearance $(\mathrm{CrCl})$ is equal to at least $50 \mathrm{ml} / \mathrm{min}$ and $15 \mathrm{mg}$ daily for $\mathrm{CrCl}<50 \mathrm{ml} / \mathrm{min}$. For all other indications, rivaroxaban should be avoided if $\mathrm{CrCl}<30$ $\mathrm{ml} / \mathrm{min}$. The most common reason for a rivaroxaban order (Figure 3) was dose-indication mismatch, followed by incorrect dose based on renal function, and then duplication.

Figure 2.

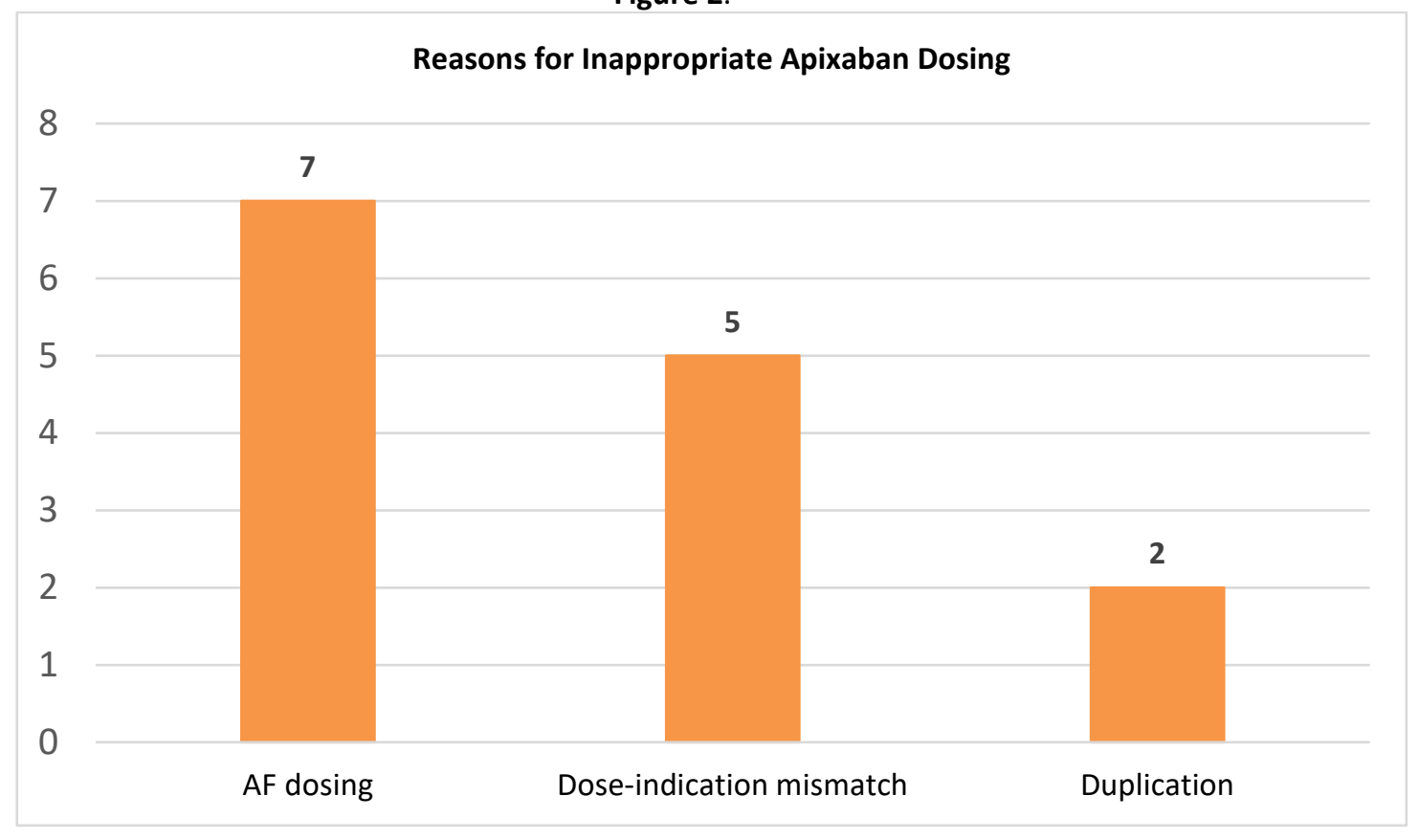


Figure 3.

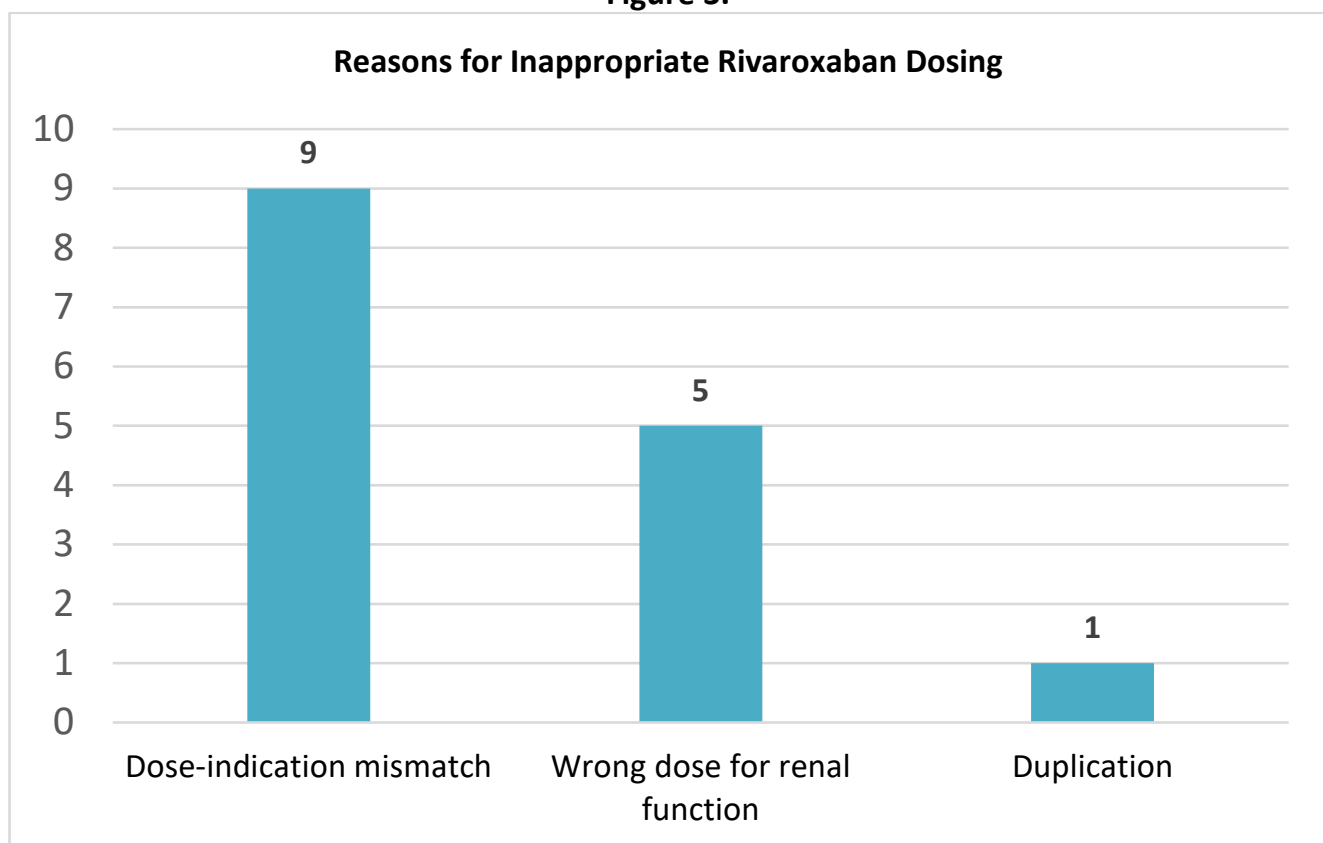

*Numbers exceed 13 because some orders were inappropriate for more than one reason.

Figure 4 highlights the results of the safety and effectiveness outcomes. Only 7 patients total experienced a major bleed event. Three of these patients were on apixaban and 4 on rivaroxaban. Of the 7 total events, only 1 could be attributed to the dose being too high, and only 1 could be attributed to duplication with another anticoagulant. Three patients experienced thromboembolic events, and none were attributed to an inappropriately low dose.

Figure 4.

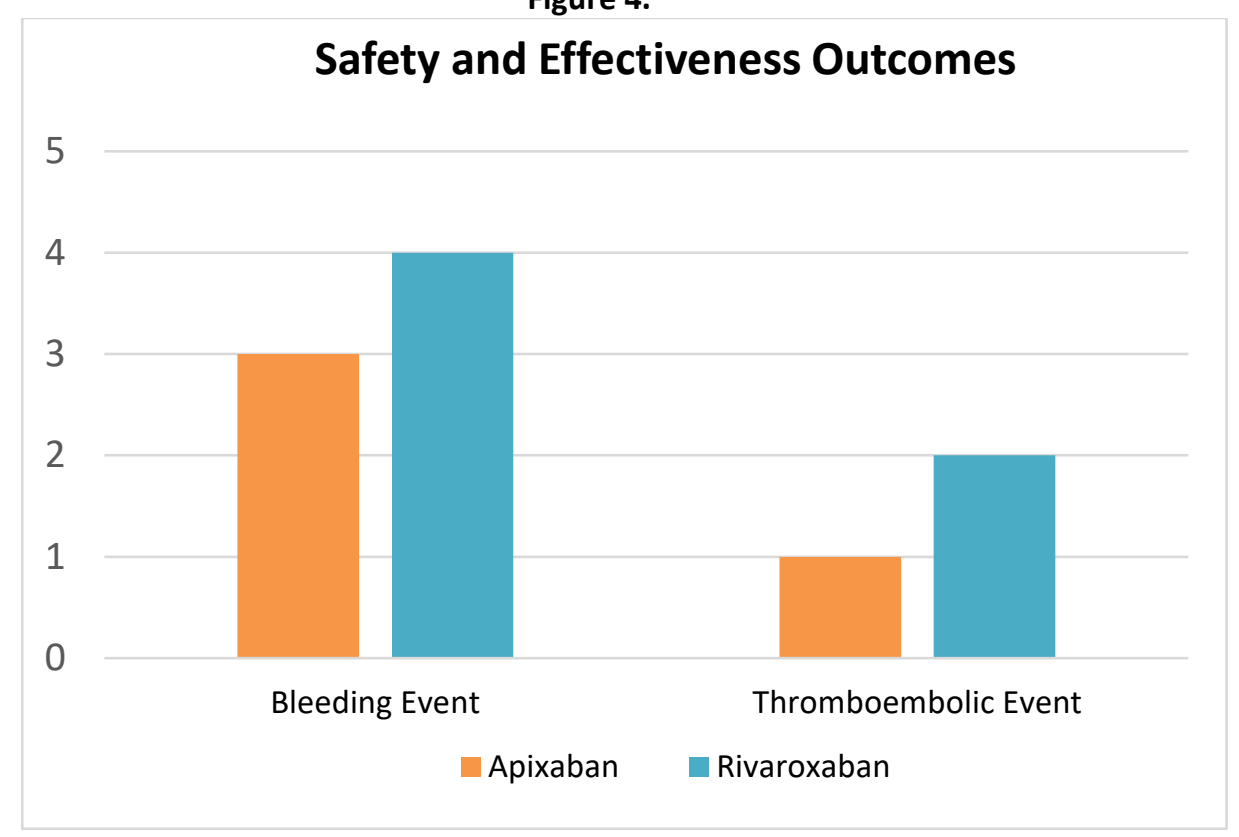

Figure 5 highlights the pharmacist clinical intervention review. There was a total of 30 pharmacist clinical interventions on DOAC orders that were documented during the 6-month period. The authors suspect that the true number of pharmacist clinical interventions on these agents was actually higher since pharmacists often forget to document such interventions. The most common reason for a pharmacist intervention on a DOAC order was duplication with another anticoagulant, followed by dose-indication mismatch, and then incorrect AF dosing for apixaban. 
Figure 5.

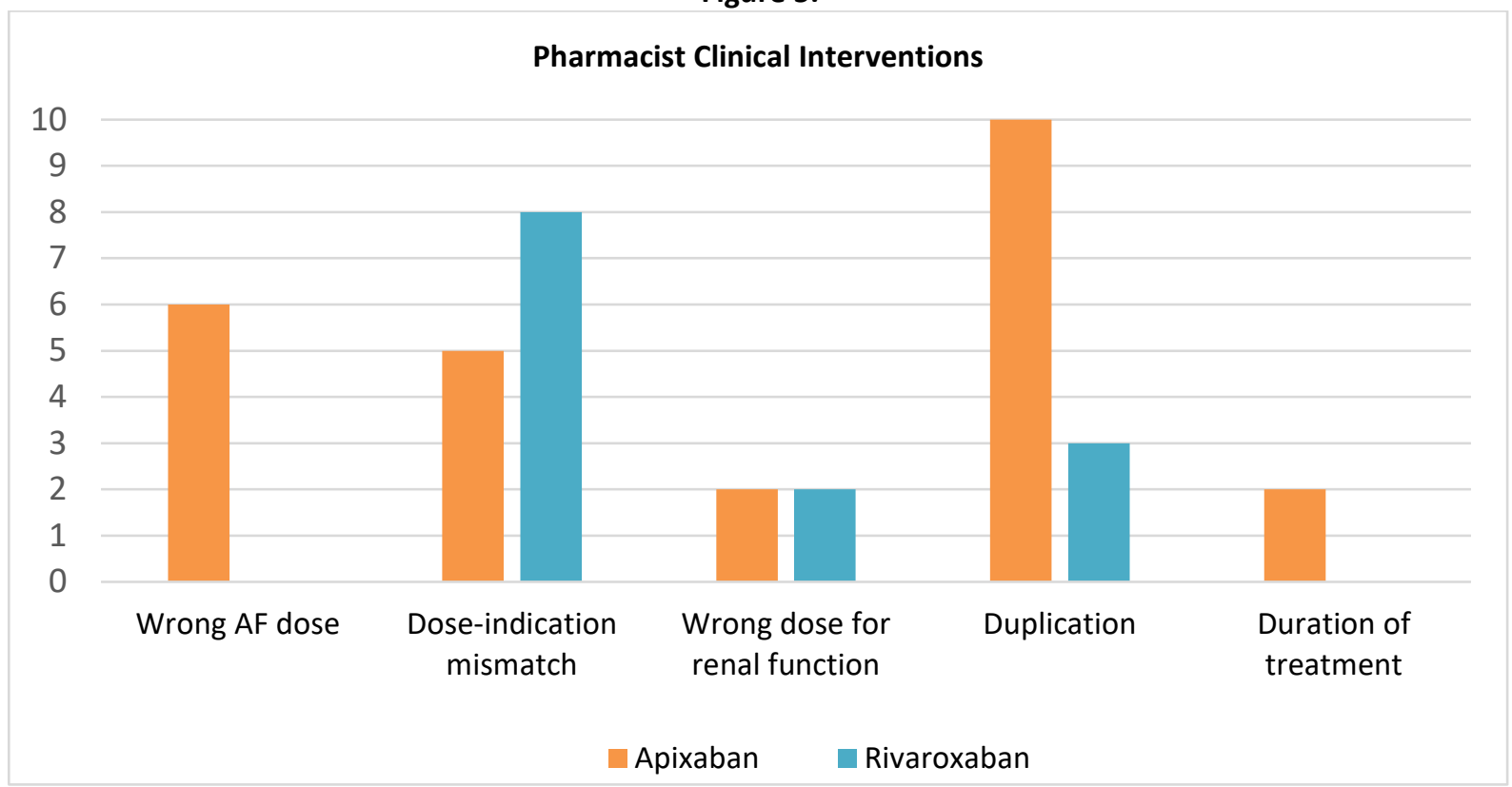

\section{Discussion}

Because DOACs have become very popular choices for the treatment and prevention of thromboembolic events, it is important to ensure appropriate use of these agents. The purpose of this study was to assess the appropriateness of DOAC orders at a community hospital, understand the implication of inappropriate orders on safety and effectiveness, and track pharmacists' clinical interventions on these high-risk medications.

The findings of this study suggest that inappropriate DOAC orders are missed by pharmacists at least $27 \%$ of the time. Since this number did not include orders that were intervened on by a pharmacist, the true percentage of inappropriate DOAC orders as a whole is suspected to be higher. The authors also found that regardless of dose appropriateness, there was a very low rate of bleeding and thromboembolic events overall. However, this is not consistent with other studies which have found higher rates of bleeding and thromboembolic events related to incorrect dosing regimens with the DOACs [3]. This inconsistency is likely due to the small sample size in the current study. In addition, patients who did experience an event may not have returned to $\mathrm{DCH}$ for care and were therefore lost to follow-up.

Lastly, the authors found that there was an average of 5 pharmacist clinical interventions per month related to the DOACs included in this study. While this number seems low, it is important to note that interventions were only captured if they were documented. Therefore, there could have been additional interventions that were missed due to lack of documentation. The most common reason for pharmacist clinical intervention was duplication. This is likely attributed to the fact that the order verification system flags duplicate anticoagulant orders at the point of verification. However, there is no way to flag an order for appropriateness based on indication, which is likely the reason interventions are lower in this category.

In addition to the ones previously mentioned in the above paragraphs, there are several additional limitations to this study that need to be addressed. One such limitation, that was alluded to previously, is not being able to determine an overall percentage of inappropriate orders due to the way the system reports had to be generated. Unfortunately, there was no way to run a single report that included both original orders and pharmacist clinical interventions in order to capture a more realistic picture of the DOACs. This type of review also leaves room for errors in data collection and relies heavily on proper chart documentation, which is often inadequate.

Despite these limitations, the authors believe this review highlights that there is still room for improvement when it comes to correctly dosing the DOACs. At a minimum, education should be provided to both physicians and pharmacists at $\mathrm{DCH}$ to address current issues surrounding this topic. Another suggestion would be to include a required indication field on the order that physicians must acknowledge when ordering a DOAC. This process would allow pharmacists to more easily and efficiently recognize inappropriate dosing regimens based on the indication for which the drug is being ordered rather than having to dig through potentially unreliable documentation in the patient chart. A final suggestion would be to consider a pharmacist led DOAC program such as the one described by Miele et al [4]. 


\section{Conclusions}

There is still a high prevalence of inappropriate DOAC prescribing that may be overlooked by both physicians and pharmacists, particularly in the inpatient setting. Education should be provided to both prescribers and pharmacists to ensure safe and efficacious therapy with the DOACs.

Conflicts of Interests: We declare no conflicts of interest or financial interests that the authors or members of their immediate families have in any product or service discussed in the manuscript, including grants (pending or received), employment, gifts, stock holdings or options, honoraria, consultancies, expert testimony, patents, and royalties.

Treatment of Human Subjects: IRB review/approval required and obtained

The opinions expressed in this paper are those of the author(s).

\section{References}

1. January C.T.; Wann L.S.; Calkins H.; et al. 2019 AHA/ACC/HRS Focused Update of the 2014 AHA/ACC/HRS Guideline for the Management of Patients With Atrial Fibrillation: A Report of the American College of Cardiology/American Heart Association Task Force on Clinical Practice Guidelines and the Heart Rhythm Society in Collaboration With the Society of Thoracic Surgeons. Circulation. 2019;140(2).

2. National Patient Safety Goals. The Joint Commission. 2019

3. Yao X.; Shah N.D.; Sangaralingham L.R.; et al. NonVitamin K Antagonist Oral Anticoagulant Dosing in Patients with Atrial Fibrillation and Renal Dysfunction. J Am Coll Cardiol. 2017;69(23):2779-2790.

4. Miele C.; Taylor M.; Shah A. Assessment of Direct Oral Anticoagulant Prescribing and Monitoring Pre- and Post-Implementation of a Pharmacy Protocol at a Community Teaching Hospital. Hosp Pharm. 2017;52(3):207-213.

5. Eliquis [package insert] Princeton, NJ: Bristol-Myers Squibb Company; 2019.

6. Pradaxa [package insert] Ridgefield, CT: Boehringer Ingelheim Pharmaceuticals, Inc; 2018.

7. Savaysa [package insert] Parsippany, NJ: Daiichi Sankyo, Inc; 2015.

8. Xarelto [package insert] Titusville, NJ: Janssen Pharmaceuticals, Inc; 2019.

9. Schulman S.; Kearon C. Subcommittee on Control of Anticoagulation of the Scientific and Standardization Committee of the International Society on Thrombosis and Haemostasis. Definition of major bleeding in clinical investigations of antihemostatic medicinal products in non-surgical patients. J Thromb Haemost. 2005;3:692-4. 